\title{
Die Besonderheiten der zivilprozessualen Kommunikation
}

Die Ziviljustiz ist immer noch eine stille Gewalt. Es gibt zwar mebr Selbstdarstellung, auch bei den Instanzgerichten, insbesondere durch Gerichtspräsidenten und Sprecher. Die Öffentlichkeit ist aber in den Prozessen selten vertreten und erfährt in der mündlichen Verhandlung oft kaum etwas von den Konflikten im Herzen der Zivilgesellschaft. Die Urteilsveröffentlichungen nebmen zu. Wie die Urteile zustande kommen, bleibt aber verdeckt. Deswegen wendet sich der Beitrag den Profilen der Akteure des Zivilprozesses und ibren Beziebungen untereinander zu. Seine Betrachtungen, auf richterliche Eigenerfabrung gestützt, sind zugleich Vorschläge für das Programm des neuen Fachs Rechtspsychologie.

\section{Einleitung ${ }^{1}$}

Der Zivilprozess war bislang praktisch kein Gegenstand der Rechtspsychologie, wenn man vom Familienrechtsstreit und der Zeugenpsychologie absieht, wobei letztere allerdings meist ebenfalls nicht speziell dem Zivilrechtsstreit gilt. Vor dem Zivilrichter entfalten sich die ökonomischen und sozialen Konflikte der Zivilgesellschaft im Detail, Konflikte, die nicht immer eine wesentliche psychische Dimension haben, aber sehr oft eine verdeckte. Umgekehrt entstehen Konflikte auch fern von jeder geistig-seelischen Wurzel, sei es, dass die Ursache eines Baumangels gesucht, das Ausmaß eines Schadens festgestellt, zur Vermeidung einer Insolvenz eine Zahlungspflicht bestritten oder ein Vertragsprogramm durch die technische Entwicklung überholt wird. Wie aber der Rechtsstreit geführt und vor allem welche Lösung hier gefunden wird, ist fast stets von der Art und Weise der menschlichen Kommunikation und der psychischen Verarbeitung der Beteiligten abhängig.

Andererseits begegnen uns etwa bei den Familiengerichten als sozialwissenschaftlich besonders gefordertem Teil der ordentlichen Gerichtsbarkeit emotional aufgeladene Konflikte um Kinder, Küche und Kabalen, deren ökonomische und soziale Ursachen die Parteien oft psychologisch ausblenden, so dass am Ende mancher befreiungspsychologisch aufgeladene Scheidungspartner sich mit einer ungeahnten Bürde auf den weiteren Lebensweg machen muss. Da es dafür aber interdisziplinäre Untersuchungsansätze angibt, wende ich mich dem Zivilprozess mit unausgewiesener psychologischer Dimension zu. Dafür kann ich die Erfahrung von über zwei Jahrzehnten richterlicher Praxis einbringen, an der ich als Professor des Privatrechts in einem richterlichen Amt bei Landgericht und Oberlandesgericht mit kleinem Dezernat beteiligt war. Dabei war ich keineswegs nur teilnehmender Beobachter, sondern habe mich, ohne die Abstumpfung durch tägliche Routine, in die Rechtsanwendung eingemischt. Der Beitrag beschränkt sich somit auf die instanzrichterliche Praxis, da die höchstrichterlichen Kommunikations- und Wirkungszusammenhänge grundsätzliche Unterschiede aufweisen. Aus der Praxis der Instanzgerichte lässt sich aber

I Der Aufsatz ist Teil eines Vortrags, den der Verfasser in einer Vorlesungsreihe zur Rechtspsychologie im Wintersemester 2003/2004 an der Universität Bremen gehalten hat. 
nicht nur Material aufbereiten, das für die rechtspsychologische Forschung von

Bedeutung sein kann, sondern eine komplementäre Strukturierung der richterlichen Innen- und Außenkommunikation in Angriff nehmen. Die Grundfragestellung, inwieweit psychologisch relevante Fakten und Abläufe die Rechtsfindung determinieren, soll dabei mit einer Vorstellung des Personals (dazu II) und der Beziehungsstrukturen (dazu III) bearbeitet werden, mit dem Ziel, die Kategorien einer Psychologie rechtlicher Befriedung auszudifferenzieren.

\section{Das Personal}

Sieht man sich die Karikaturen von Richtern, Staats- und Rechtsanwälten in der bildenden Kunst von Hogarth über Daumier bis Th. Th. Heine an, dann begegnet man einer Galerie ausdrucksloser Eulengesichter, knöcherner Besserwisser, aufgeblasener Bramarbasierer, lebensfremder Korinthenkacker, abgrundtief Gleichgültiger und eitler Posierer mit Omnipotenzgehabe. ${ }^{2}$ Stets scheint danach die Justiz der Gesellschaft einige Jahrzehnte hinterherzuhinken, so wie die Weimarer Justiz weitgehend monarchistisch und antidemokratisch war und die der fünfziger Jahre nichts wissen wollte vom vorausgegangenen selbstverschuldeten Unheil der deutschen Geschichte.

\section{Die Richterschaft}

Heute ist hingegen die Richterschaft nach dem kulturellen Umschwung von I 968 viel pluraler. Es gibt ihn zwar noch, den menschenscheuen Aktenleser, der möglichst keine Zeugen sehen, sondern alles den Papieren entnehmen will. Es gibt ihn in der gründlichen Variante, wo alles Wort für Wort gelesen und immer wieder ergänzt wird, wie in der fixen, wo jeder nicht sofort perfekte, sondern erst nach und nach vertiefende Vortrag als verspätet zurückgewiesen wird. Es gibt noch den entscheidungsschwachen Richter, der keinem weh tun will, vieles durch Aussitzen erledigt, die Beteiligten bis zur Zermürbung anhört und vernimmt und alles durch Vergleich lösen will. Aber abgesehen von diesen Grenzfiguren ist die Ziviljustiz heute überwiegend eine Schreibbürokratie, die aber doch in Verhandlungen kommunikativ auf eine Einigung der Parteien drängt, im historischen Vergleich sehr zügig arbeitet, die Jüngeren stets auch mit digitaler Autonomie. Dabei ist das Vorbild, dass ein Richter in seinem Text möglichst keine offenen argumentativen Angriffsflächen bietet, den Parteien gegenüber wirtschaftlichen Durchblick entwickelt und auch für die soziale und psychische Situation der Beteiligten zumindest Verständnis entfaltet. Er muss möglichst einem Interview für die Öffentlichkeit gewachsen sein. Für seine Karriere ist aber das Wesentlichste, dass keine Aktenrückstände entstehen. ${ }^{3}$ Im Zivilprozess geht es einerseits um Banalitäten und Marginalitäten von Reparaturmängeln bis Nachbarstreitigkeiten, aber auch um Existentielles, so wenn eine Berufsposition oder der Bestand

2 Siehe insbesondere Cornelis Veth, Der Jurist in der Karikatur, Reprint der Ausgabe Berlin 1927, Fachhochschulverlag, Frankfurt a. M. 2003.

3 Bei meiner eigenen richterlichen Tätigkeit habe ich sowohl Persönlichkeiten kennengelernt, die das Format zu weitreichender gesellschaftlicher Gestaltung und einen tieferen Bildungshintergrund hatten, wie auch das gesamte Spektrum akademischer Berufstätigkeit mit seinen großen Begabungsdifferenzen anzutreffen war. 
eines kleinen Unternehmens auf der Kippe steht. Hier darf der Richter keinen Überdruss erkennen lassen, was aber älteren, nicht mehr beförderungsfähigen Richtern teilweise nicht mehr gelingt.

Der Hauptdefekt jeder Bürokratie, die Tendenz zu Passivität, Wiederholung und Faulheit, pardon: Antriebsschwäche, kann in der Richterschaft gut camoufliert werden. Die Akten nicht gelesen, ohne sachliches Interesse, mit vagen Vorstellungen über den Streitgegenstand - das schließt es für einen Richter nicht aus, als weiser Salomon aufzutreten, beiden Parteien Verständnis zu bekunden und die hälftige Teilung anzuregen. Das ist eine in den Folgen verheerende Haltung. Die Anwälte, die mit diesem pseudohumanen Salomonismus rechnen müssen, können sich einen differenzierten Vortrag schenken und müssen, strategisch gesehen, die Forderungen ihrer Mandanten von vornherein auf Kosten eines sachgerechten Vortrags aufblähen und damit Anwalts- und Gerichtskosten so ausweiten, dass der Prozess leicht ganz unökonomisch wird, selbst bei noch so berechtigtem Forderungsgrund. Wer verklagt ist, wird praktisch gezwungen, sofort Widerklage in gleicher Höhe zu erheben, um seinen Mandanten ganz vor dem Klagbetrag zu verschonen. Paradoxerweise ist damit der Salomonismus eine der unerträglichsten Pathologien des Zivilprozesses. Am Ende eines derart durch Klage und Widerklage ausgeschöpften Streitrituals bleiben im zugespitzten Fall beide Klagen erfolglos und jede der Parteien trägt die Hälfte der Gerichtskosten sowie die Kosten des eigenen Anwalts. Das ist dann die Strafe für die Inanspruchnahme von Rechtsschutz. Diese kann auch noch verschieden hoch ausfallen, je nachdem, wer den bei der Honorarbeitreibung kulanteren Anwalt genommen hat. Insofern zeitigt Salomonismus die Höchststrafe für die Befassung der Justiz. In reiner Form ist dieser Typus glücklicherweise noch nicht durchgehend verbreitet; er ist aber nicht ganz selten.

Von den sonstigen Fehlformen neuerer justizieller Bürokratie möchte ich wenigstens noch zwei nennen, den Nischenakribiker und den Akzeleristen. Bekanntlich schreitet die Verrechtlichung der Gesellschaft ungeachtet aller Warnungen stetig voran. Der Grund liegt darin, dass die theologischen, ethischen und moralischen Wertungskonsense in einer säkularisierten multikulturellen Gesellschaft immer mehr verblassen und das Recht insofern Auffangfunktionen zu erfüllen hat. Das führt zu einer permanenten Ausdifferenzierung von Rechtsmaterien und Rechtsprechungsbeständen zum nationalen, europäischen und internationalen Recht. Den Überblick zu behalten, gelingt fast nur noch von hoher und höchster Warte. Die perfekte Detailsicherheit ist praktisch nur in Einzelbereichen und Nischen möglich. Es gibt den Markenrechtler, der jede wichtige Markenrechtsentscheidung samt Sachverhalt aus den letzten Jahrzehnten kennt, sonst aber praktisch nur juristisches Zeitungswissen hat, und den Arzthaftungsrechtler, dem schon bei der Architektenhaftung schwummrig wird. Im Richterberuf, in dem die Garantie der Entscheidbarkeit jedes Falls besteht, ${ }^{4}$ zieht sich der auf umfassendes Wissen bauende Perfektionist gern in eine Nische zurück, wo er sich restlos sicher fühlt, und die Arbeitsteilung bei den höheren und höchsten Gerichten begünstigt dies. Die Nischen werden auch wegen der internen Stoffexpansion immer kleiner. Nun befindet sich allerdings das Recht in den entwickelten Wirtschafts- und Rechtsordnungen in einem ständigen Anpassungsprozess, den zu begreifen aus der Nische heraus kaum oder schwer möglich ist. So kann sich hier umfassendes Nischenwissen sehr pointiert mit gesamtgesellschaftlicher Ahnungslosigkeit verbünden. Der Begriff Fachidiot ist dafür nicht zureichend, weil es nicht nur um die Beschränkung durch den Fachhorizont geht, 
sondern um die äußerste Gewissenhaftigkeit im Fachlichen. Der Beruf des Richters, der notfalls am Ende entscheidet, verleitet aber auch dazu, die Fachgewissheit ins Fachfremde zu verlängern. Nischenakribiker gibt es zwar auch in Wissenschaft, Verwaltung und Technik. Aber diese haben wenigstens nicht während ihrer gesamten Berufszeit das letzte Wort.

Der Akzelerist kann sich auf die beiden großen Novellen der Zivilprozessordnungs stützen, deren Hauptanliegen die dem Tempo des entwickelten Kapitalismus Rechnung tragende Beschleunigung war. Ewig lange Zivilprozesse waren deutsche Rechtstradition, mit dem Wetzlarer Reichskammergericht als groteskester Aktenliegenschaftsverwaltung. Noch in den sechziger Jahren des letzten Jahrhunderts war etwa das Anwaltskartell eine verbreitete Unsitte, bei dem in den Instanzen Prozesse aufgerufen wurden, die für den jeweiligen Tag eingeteilten Anwälte aus den beim Gericht aufgehäuften Akten Anträge stellten, ohne die Akten zu kennen, die Parteien nicht zugegen waren und die Richter etwaige Fragen in Beschlussform stellen mussten. Damit hat die Vereinfachungsnovelle von 1976 aufgeräumt, wenn man von Ausnahmefällen wie dem Landgericht Düsseldorf absieht, wo es offenbar seither keinem Präsidenten gelungen ist, das Kartell abzuschaffen, obwohl doch die Düsseldorfer Richter- und Anwaltschaft auch kaum so viel schlafmütziger sein dürfte als diejenige in anderen Regionen. Die Novelle des Jahres 200I, durch die Justizministerin Däubler-Gmelin erzwungen, hat die erste Instanz zum entscheidenden Gericht machen wollen, was prompt die Berufungsinstanzen in erheblichem Umfang zu Ruhezonen gemacht hat, wo allzu gern die Rechtsmittel ohne weiteren Zusatz »aus den zutreffenden Gründen« der ersten Instanz zurückgewiesen werden und das Unwesen einstimmiger unverzüglicher Zurückweisungsbeschlüsse ohne mündliche Verhandlung um sich greift. ${ }^{6}$ In jedem Fall ist der Richter, der ganz schnell entscheidet, jetzt als gesetzliches Leitbild verankert, zumal die staatlichen Sparzwänge ein stetiges Anwachsen des Pensums bewirkt haben. Bei einem Richter, der eine Rechtssache nicht entsprechend schneller analysiert, beschränkt sich das Argumentationsarsenal daher sehr leicht auf vier Muster: verjährt, verwirkt, versäumt, verspätet! Damit lassen sich auch komplexe Streitsachverhalte simplifizieren. Verspätungsfetischisten gibt es inzwischen bei jedem größeren Gericht. Der Akzelerist ist ein Richter, der entschlossen zu Lasten der Qualität entscheidet, dem also Tempo vor Gerechtigkeit geht.

Die deutsche Ziviljustiz spiegelt aber einschließlich dieser Prototypen zur Zeit das politische Spektrum der Gesellschaft im großen und ganzen wider. Zwischen Marktradikalismus und Schutzstrategien zugunsten von Verbrauchern, Patienten, Mietern, kleinen Mittelständlern und anderen Gruppen von Schwächeren sind alle Nuancen vorhanden. Die Einfühlungsbereitschaft ist aber regelmäßig größer, wenn marktkritische Grundhaltungen erkennbar sind.

5 Siehe das Gesetz zur Vereinfachung und Beschleunigung gerichtlicher Verfahren vom 3. I 2. I 976, in Kraft getreten am I. 7. 1977 (BGBl. I, 328I), und das am I. I. 2002 in Kraft getretene Gesetz zur Reform des Zivilprozesses vom 27.7. 200 I (BGBl. I, I 887).

6 Siehe dazu \522 II ZPO, wo der einstimmigen Entscheidung zur Aussichtslosigkeit der Berufung der Test in mündlicher Verhandlung erspart wird. Der Gesetzgeber hat dabei verkannt, dass der einstimmige Beschluss in den Kammern und Senaten die Regel ist. 
In der Anwaltschaft ist der Facettenreichtum noch viel größer als in der Richterschaft. Da schon seit langem der Großteil der Juraabsolventen, die in juristische Berufe gelangen, den Anwaltsberuf ergreift, gegenwärtig 80\%, ist dort jede erdenkliche Spezies anzutreffen, was juristisches Know-how, Verhandlungsgeschick, wirtschaftlichen Durchblick, soziale und psychologische Kompetenz angeht, aber auch die Bereitschaft, das Mandanteninteresse über das Eigeninteresse zu stellen. Bis zu einem Betrag von über I0.000€ ist es in jedem Fall wegen der Gerichts- und Anwaltskosten unwirtschaftlich, einen Prozess über zwei Instanzen zu führen, wenn man ihn nicht (was selten ist) restlos gewinnt und der Kontrahent dann auch leistungsfähig ist. Anders ist es nur, wenn man rechtsschutzversichert ist oder als Minderbemittelter Prozesskostenhilfe erhält. Die Typen der historischen Anwaltskarikatur gibt es zumindest ansatzweise noch heute, den Heißlufterzeuger, den Wortverdreher, denn Allesbestreiter, den, der ewig Ruhe ausstrahlt, und den, der die friedlichsten Parteien in Rage bringt. In den Fabriken mit Hundertschaften von Anwälten entwickelt sich der Spezialist, der dringend andere Spezialisten braucht, wenn der Fall sich (wie meist) nicht in die Spezialisierung fügt. Dann tauchen bei Beratung und Prozess oft kleine Mannschaften auf, die aber nicht selten vor lauter Fachwissen zu keiner Strategie zusammenfinden. Der arme Aufsichtsratsvorsitzende Breuer von der Deutschen Bank etwa, von dem Frankfurter Riesenbüro Henggeler und Müller vertreten, war für seine Aussage im Prozess gegen Kirch gewiss gebrieft worden, wie man dies bei einem hohen Herrn eben macht, der die Geschicke einer Großbank gelenkt hat, also eher devot. Dann redete dieser sich und seine Großbank um Kopf und Kragen, niemand hatte ihn vor Weitschweifigkeiten gewarnt, die den Schadensersatzanspruch Kirchs erst richtig schlüssig machten. Und keiner der hochprofessionellen Spezialisten war in der Lage, die Vernehmung mit der menschlichen und rechtlichen Souveränität zu steuern, die notwendig gewesen wäre. Ein Spezialist für Vernehmungen war eben nicht dabei.

In kleinen Büros geht es zwar persönlicher zu, oft aber auch zu persönlich. Hier wird die Partnerschaft ebenfalls nicht selten gewechselt, auch noch nach Jahren. Die Trennung ist dann oft schwieriger als eine Scheidung, weil jedes Mandat und jeder gewonnene Klient einschließlich der künftigen Beratungsgebühren und Prozesse verteilt werden müssen. Besondere Nähe der Zusammenarbeit intensiviert dann auch die Trennung. Die Mandanten wissen dann oft nicht mehr, an wen sie sich halten sollen und bei wem ihre Daten alles landen. ${ }^{8}$

Auch der Einzelanwalt ist eine vielgestaltige Spezies. Da gibt es denjenigen, der für seine treuen Mandanten alles macht, vom Verkehrsunfall über die Vorfälligkeitsentschädigung bis zum Umgangsrecht mit den Kindern und zur Zusatzrente und sich jeweils notfalls recht gründlich einarbeitet. Andere nehmen nur Mandate aus dem Internet von Anlegern, die aufgrund zweifelhafter Bankberatung ihr Geld verloren haben, oder betreuen die Mieter ihres Viertels bei Mieterhöhung und Kündigung. Der Feld-Wald-Wiesen-Anwalt macht, wenn er gut ist, alles auf einfachem Niveau, aber keineswegs erfolglos. Aber es kann auch ein Unglücksmensch sein, der seine Mandanten regelmäßig ins Chaos reißt, und das oft mit hohem Einsatz. Nicht zu vergessen ist auch der Abzocker, der unnötige Prozesse vom Zaun bricht, die Streitwerte hochtreibt, die Instanzen ausschöpft und seinen Mandanten notfalls Haus und

8 Die Verschwiegenheitsverpflichtung der Anwälte nach $\$ 49$ b IV I BRAO behindert die Rechtsgeschäfte über Anwaltspraxen und Honorarfragen und wird dementsprechend massiv kritisiert und relativiert (s. etwa Prechtel, NJW 1997, I8 I 3; Paulus, NJW 2004, 21). 
Hof wegen der Kosten verlorener Prozesse wegpfändet. Das Büro solcher Anwälte ist dann auf die Abwehr von Attentaten getrimmt. Die Anwesenheit des Anwalts wird hier nur noch bestätigt, wenn die Gefahr von Beschwerden oder Angriffen gänzlich ausgeräumt ist. »Nicht im Haus« oder »in einer Besprechung « heißt dann das dauerhafte Motto. Selbst in einer nüchternen Großstadt wie Bremen gibt es für die verschiedenen Typen in Justizkreisen entsprechende Spitznamen. Wenn wir - zur Vermeidung von Prozessen - den ehrenhaften Allerweltsnamen Müller exemplarisch dafür missbrauchen dürfen, dann gibt es etwa Dröhn-Müller, Gebühren-Müller oder Zeugen-Müller, der sich selbst immer als Zeugen benennt, und sogar Box-Müller, der sich physisch zu wehren weiß.

\section{Die Parteien}

Die Parteien entziehen sich einer vergleichbaren Kategorisierung. Es gibt Prozesse, da steht wirklich auf der Kippe, wer Recht behält. Als Richter kann es einem passieren, dass man immer dem Recht geben möchte, der zuletzt gesprochen hat. Dann gibt es eine Klientel, die, ohne querulatorisch zu sein, die ordentlichen Gerichte überproportional beschäftigt, sei es wegen Rechthabertums oder Überambitioniertheit. Lehrer und Zahnärzte haben als Bauherren insofern bei der Richterschaft einen schlechten Ruf, der Lehrer wohl pars pro toto für Beamte, die sich viel Zeit zur Einarbeitung in die für sie maßgebliche Rechtsmaterie nehmen, aber dabei völlig auf ihr Eigeninteresse fokussiert sind. Viele Prozesse verdanken sich der Zahlungsunfähigkeit einer Partei, die das nicht zugibt, womöglich auch sich selbst nicht, und deswegen alle denkbaren Gegenrechte geltend macht, um einen vollstreckbaren Titel hinauszuschieben. Am schlimmsten ist es, wenn sowohl der Kläger wie der Beklagte einen Prozess zur Sanierung ihrer Existenz brauchen. Unfreiwillig oft beim Gericht sind die Pfuscher, sowohl aus dem handwerklichen wie aus dem freiberuflichen Bereich. Die beim Zivilgericht verbrachte Zeit muss für jeden unternehmerisch denkenden Menschen ökonomisch und sozial frustrierend sein. Daraus ergibt sich ein sachlicher Einigungszwang, der insbesondere Referendare und junge Berufspraktiker meist überrascht. Trotz größter und ausgebreiteter Zerstrittenheit kommt es deswegen oft noch nach zwei oder drei Instanzen zu einer Einigung der Parteien, für die das Gericht geeignete psychische Dispositionen fördert, indem es den Parteien beste Persönlichkeitsprofile zubilligt. Hochherzigkeit, Mitmenschlichkeit, Souveränität, Selbstkritik, Realitätssinn, Entschlossenheit und Professionalität vereinen sich so oft für eine Minute der Harmonie zu einer vergleichsweisen Regelung der Kontrahenten, auch wenn die Parteien dann schon beim Verlassen des Gerichts ihre dunklen Seiten wieder stärker in sich spüren und zunehmend von Reuegefühlen übermannt werden.

\section{Die Zeugen}

So bunt wie die Parteien erscheinen auf den ersten Blick die Zeugen gemischt. Dabei handelt es sich aber um eine von den Parteien und ihren Anwälten meist wohlbedachte Auswahl. Am besten benennt man einen wirtschaftlich oder sozial abhängigen Zeugen, der ein elementares Interesse daran hat, dass seine Partei den Prozess gewinnt und er seinen Beitrag zur Erhaltung des Betriebsfriedens oder gar seines Arbeitsplatzes leisten kann. Bei einem Rechtsstreit eines Unternehmens sind dies dessen 
Angestellte, die denen des gegnerischen Unternehmens oder dem prozessierenden Konsumenten gegenübertreten. Der Verbraucher kann oft allenfalls seinen Ehepartner oder Lebensgefährten aufbieten. Hier reproduziert sich dann auf der Beweisschiene das Machtgefälle des wirtschaftlichen Verkehrs sehr leicht. Ein Beispiel: Ein Handwerker hat aufgrund eines telefonischen Schnellauftrags eines Bauunternehmens zwanzig Fenster angefertigt, die dann in den Maßen nicht passen, sondern zu klein sind. Drei Mitarbeiter des Bauunternehmens, einer $\mathrm{GmbH}$, stehen als Zeugen bereit, um die Vorgabe der richtigen Maße als Mithörer des Telefongesprächs zu bestätigen, desgleichen die Kenntnis des Handwerkers von dieser Mithörerschaft. ${ }^{9}$ Der Handwerker ist Partei und kann nicht Zeuge sein. Seine Ehefrau kann als Zeugin noch nicht einmal auf eine Mithöreinrichtung in ihrem Haus verweisen und als Fachfremde höchstens Wahrnehmungsfragmente authentisch darlegen. Der Mächtige tut sich also mit dem Beweis grundsätzlich leichter. Es gibt zwar keine archaischen formellen Beweisregeln mehr wie den Satz »Dreier Zeugen Mund tut die Wahrheit kund«. Der Richter hat das Privileg der freien Beweiswürdigung, kann also auch einem zufälligen Passanten in einem Verkehrsunfallprozess Glauben schenken, während drei Polizisten das Gegenteil sagen. Zu bedenken ist nur, dass ein Urteil, in dem ein Zeuge als unglaubwürdig, eine Aussage als unglaubhaft bezeichnet wird, einfach viel aufwändiger ist als die Akzeptanz für das, was jeder Zeuge aussagt. Die richterliche Haltung, die praktisch jedem Zeugen Glaubwürdigkeit unterstellt, ist daher nicht ohne weiteres das Zeichen eines besonders positiven Menschenbildes, sondern auch einer bürokratisch unaufwändigen Erledigung. Aber dies berührt schon die Beziehungsstrukturen, auf die noch genauer einzugehen ist.

Zeugen aus Institutionen sind regelmäßig nicht aufgrund von Interessenübereinstimmung prädisponiert, sondern als Akteure einer Recht, Gesetz und Behördenaufgabe genügenden Amtsführung. Der Kommunalbeamte etwa, der nichts von der Bodenverunreinigung des verkauften Kommunalgrundstücks gewußt hat, nimmt das Amtsinteresse wie sein eigenes wahr. Dabei ist die Konformität der Aussagen regelmäßig erhöht, da die vorprozessuale Wahrheitsfindung zur Amtsaufgabe gehört.

Neben den Interessenzusammenhängen des Marktes und seiner Teilnehmer und den Selbstdarstellungszwängen der Institutionen sind im Zivilprozess die Familienkohärenzen von besonderer Bedeutung, da Verwandte und Angehörige das private Geschehen am ehesten begleiten. Dass ein Beweis über das Zeugnis des Ehegatten möglich ist, überrascht immer noch viele Laien, wahrscheinlich aufgrund eines idealisierten Familienbildes. Während aus der etwas angeschlagenen Kernfamilie, den Alleinerzieher- und Singlehaushalten allerdings zunehmend spärlicherer Beweis zu schöpfen ist, verfügen ausländische Großfamilien als intakte Solidarverbände vor dem Richter über mehr Beweispotential. Da kann schon eine zehnköpfige Zeugenkohorte einer türkischen Familie zum Beweis dafür angeboten werden, dass der Preis für den gekauften Obst- und Gemüseladen von ı0.000€ am Freitagnachmittag in einem bestimmten Café ohne Quittung in Scheinen geflossen ist, während die Verkäuferfamilie ebenso viele Zeugen dafür aufbieten kann, dass gar nichts beglichen worden ist. Die Zeugen wären vermutlich jederzeit zum Eid bereit, wenn der Richter einen Schwur wollte. Der deutsche Zivilrichter will aber solche Zeugen regelmäßig nicht einmal sehen, geschweige denn vernehmen. Er erklärt den Parteien dann, dass er vermutlich allen glauben wird, dass dann aber nichts bewiesen ist, es also auf die Beweislast ankommt, der Schuldner also die Zahlung beweisen muss und deswegen

9 Das BVerfG hat zwar den Beweis über Lauschzeugen im Zivilprozess nunmehr ausgeschlossen (NJW 2002, 3619). Ob damit aber auch der Beweis durch diese Zeugen ausgeschlossen ist, dass sie keine Lauschzeugen, sondern offene Gesprächsteilnehmer waren, wird noch zu klären sein. 
der Käufer voraussichtlich auch dann zur Zahlung verurteilt werden wird, wenn seine

Verwandten zehn Meineide auf sich genommen haben. Familiale Einsatzbereitschaft bricht sich dann an der Rationalität kapitalistischen Wirtschaftens. Das Ergebnis des Prozesses kann dann im Spektrum zwischen einer Zahlungspflicht (eventuell mit kleinerem Risikoabschlag) und dem Verzicht auf das Geschäft (eventuell mit kleinerer Abfindung hierfür) liegen.

\section{Die Sachverständigen}

Eine äußerst spezielle forensische Population sind die Sachverständigen. Diesen wird von den Richtern wie von der sonstigen Gesellschaft im allgemeinen große Autorität zugewiesen. Maßgeblich ist dafür aber nicht so sehr der Glaube an die jeweilige Wissenschaft, sondern die bürokratische Entlastung, die ein Sachverständiger bedeutet. Ein Sachverständigengutachten zu erschüttern und eine weitere Beweisaufnahme mit einem möglicherweise besseren Gutachter zu erreichen, ist vor deutschen Zivilgerichten extrem schwierig. Eine Partei, die gegen ein Sachverständigengutachten vorgehen will, braucht regelmäßig ihrerseits vielfach zusätzliche private sachverständige Hilfe, um etwa bei der Anhörung des Gerichtssachverständigen die richtigen Fragen stellen und eventuelle Ungenauigkeiten und Widersprüche des Gutachters herausarbeiten zu können. Im Übrigen können die Gerichte, soweit sie nach ihrer Zusammensetzung schon über Erfahrung mit den verschiedenen Sachverständigenlisten der Industrie- und Handelskammern oder anderer Institutionen verfügen, durchaus den Beweisaufwand dimensionieren.

Betrachten wir einmal den technischen Bereich, in dem die Begutachtung auf den ersten Blick weniger von sozialen Haltungen und Vorverständnissen geprägt erscheint. Aber auch hier gibt es gravierende Unterschiede. Man kann als Bautensachverständigen einen rustikalen Praktiker nehmen, der womöglich kurzfristig und entschlossen ein begründungsarmes, aber wenig angreifbares Gutachten erstattet, vielleicht auch preiswerter als andere. Häufig sind Baumängel nicht mehr mit wirtschaftlichem Aufwand zu beseitigen. Dann kann die pragmatische Haltung dieses Gutachters darauf hinauslaufen, dass ohnehin kein Bau mängelfrei ist und ein bescheidener Bauherr auch nur bescheidene Ansprüche zu stellen hat. Man kann aber auch den wissenschaftlich ambitionierten Gutachter beauftragen, der ständig wissenschaftlich publiziert, einem Baumangel bis in seine letzten Verästelungen folgt, die Ursache bis zu dem maßgeblichen ersten gedanklichen Fehler des Architekten oder Bauunternehmers ergründet, ein ausdifferenziertes Nachbesserungskonzept vorlegt und lange braucht und sehr viel kostet. Diese beiden Typen sind selbstverständlich wiederum nur Grenzfiguren. Das Gericht kann dann überlegen, ob es den besonders mäklerischen Lehrer als Bauherrn eher mit dem rustikalen oder dem hochwissenschaftlichen Sachverständigen belohnen oder bestrafen will. Den allzu wissenschaftlichen Sachverständigen wird das Gericht dann vermeiden, wenn es dessen Gutachten nicht einmal in seinen Ergebnissen richtig verstehen kann. Schließlich ist bei Richtern technisches Verständnis ein zufälliges Nebenprodukt, soweit nicht im Beruf der Aufbau entsprechender Kompetenzen gelingt. Der Richter kann sich einer Entscheidung in keinem Fall mit der Begründung entziehen, er habe das eingeholte Sachverständigengutachten nicht verstanden. Auch daraus ergibt sich das Übermaß an Akzeptanz für Sachverständigenautorität.

Dieses Übermaß an Akzeptanz ist sogar im sozialwissenschaftlichen Sektor festzustellen, auch wenn dort die große Bedeutung unterschiedlicher Vorverständnisse an 
sich klar ist. Nehmen wir ein Beispiel aus dem den Psychologen besonders erschlossenen Bereich der Familiengerichtsbarkeit, mit der ich mich selbst auseinandergesetzt habe, was die interdisziplinäre Zusammenarbeit angeht. ${ }^{\circ}$ Hier kann ich über einen gut protokollierten Fall der Begutachtung im Sorgerechtsstreit zwischen Eltern berichten, die jeweils das alleinige Sorgerecht für ihr Kind beantragt haben. Das Gericht hat sich an ein Institut gewandt, das zwei Psychologinnen in die Haushalte der getrennt lebenden Eltern geschickt hat. Beide sind auf den Besuch vorbereitet. Die Mutter hat aufgeräumt, von dem großen Hund des neuen Lebensgefährten gibt es in der Dreizimmerwohnung keine Spur mehr. Das Kinderzimmer ist in Ordnung. Es gibt gleich Kaffee und Kommunikation mit den Besucherinnen. Der häufige Körperkontakt zwischen der Mutter und dem Jungen wird eifrig registriert. Beim Vater sieht alles etwas anders aus. Er hat sich freigenommen, aber es ist doch nicht so gut aufgeräumt, auch nicht im Kinderzimmer. Die Oma, die den Jungen sonst weitgehend betreut, sagt immer, dass das eigentlich seine Aufgabe sei. Deswegen hat der Vater sie nicht dazugebeten. Die Sachverständigen fragen, was er mit dem Kind gerne macht. Basteln sagt er und wird ermuntert, das zu zeigen. Auf einen Drachen ist er vorbereitet, weil es Herbst ist. Er fängt an zu basteln, aber es soll ein besonders schöner Drachen werden. Der Junge macht zunächst interessiert mit, findet es dann aber langweilig und dreht immer wieder das Radio laut auf. Der Vater wird langsam nervös und sagt schließlich unwirsch, das Radio müsse ausgemacht werden. Die Sachverständigen sind leicht gelangweilt, und in dem Gutachten steht später: »Die ganze Zeit kein Körperkontakt.« Natürlich werden außer den Besuchen noch alle Tests durchgeführt. Beim Sonnentest ${ }^{11}$ liegt die Oma mit der Mama vorn. Sie ist aber schon 60 Jahre alt. Die Mutter bekommt das Kind. Grundlage ist das so zustande gekommene Sachverständigengutachten. Der Vater und sein Anwalt greifen es gar nicht an, sie wüßten auch nicht, wie das zu machen wäre. Dem Sachverständigen wird also - das ist das Resümee - viel zu wenig auf die Finger gesehen. Sachverständigenkritik tut not.

\section{Die Beziehungsstrukturen}

Nehmen wir Parteien, Anwälte, Richter, Zeugen und Sachverständige zusammen, so ist eine ganze Palette von Beziehungsstrukturen zu entwickeln, geradezu für ein rechtspsychologisches Forschungsmakroprogramm. Beginnen wir einmal mit den Beziehungen der Parteien untereinander.

\section{Die Beziebung der Parteien}

Zwischen den Parteien kann ein anonymes Verhältnis bestehen. Wenn etwa ein Geschädigter die Haftung eines Warenproduzenten einklagt - Stichwort zum Beispiel Kindertee und Zahnschädigung ${ }^{12}$ - wird ein Unternehmen (in seiner Produktionsmethode und seiner Produktzusammensetzung) mit einem Verbraucher konfrontiert. Die vorprozessuale Problembearbeitung mit Gutachten und Schadensfeststellung kann ganz unpersönlich verlaufen. Spätestens im Prozess wird dann jedoch eine

I० Anforderungen an eine interdisziplinäre Trennungs- und Scheidungsberatung, FuR I996, 208 ff.

I I Hier ordnet das Kind in einer Zeichnung den Personen seiner Umgebung jeweils eine Sonne zu, so dass ein Sonnenvergleich Schlüsse auf die Bedeutung für das Kind soll ergeben können.

I 2 Siehe zuletzt $B G H$, NJW 200 I, 964. 
gewisse Personalisierung unvermeidlich. Diese erzwingt ein Mindestmaß an Kooperation bei der Eruierung von Ursache und Wirkung sowie der Bestimmung der möglichen Entschädigung. Ein Unternehmensvertreter, der sich im Prozesstermin als völlig von den Weisungen seiner Firma abhängig und nicht entscheidungsbefugt darstellt, mag sich der Personalisierung zu entziehen versuchen. Das Gericht braucht dies jedoch nicht zu akzeptieren, kann schon bei der Ladung der juristischen Person als Partei einen Vertreter anfordern, ${ }^{13}$ der Entscheidungen treffen kann, und womöglich sogar während der Verhandlung Rückrufe organisieren. Die Gefahr einer ungünstigen Entscheidung bei Defiziten persönlicher Problembearbeitung zwingt somit auch die Träger eines durchorganisierten kapitalistischen Betriebs im Prozess zu einem Minimum an persönlicher Beziehungsaufnahme.

Umgekehrt können Parteien zutiefst in eine persönliche Beziehung verstrickt sein, die die sachliche Konfliktbereinigung erschweren oder unterminieren kann. Dafür braucht man nicht an Trennung und Scheidung zu denken. Schon zwischen zwei Geschäftsführern einer $\mathrm{GmbH}$, von dem der eine wagemutig investieren, der andere vorsichtig den Besitzstand verwalten will, kann der Rechtsstreit das Finale unerträglich gewordener Alltagskämpfe sein, wo jeder - womöglich jeweils aufgrund Alleinvertretungsbefugnis - die Entscheidung des anderen aufzuheben oder zu ändern bestrebt ist, und das bei beiderseitiger Abhängigkeit von produktivem gemeinsamen Wirtschaften und unentrinnbarer Pattsituation. ${ }^{14}$ Hier kann es das oberste Gebot der zivilprozessualen Kommunikation sein, dass die Parteien möglichst überhaupt nicht persönlich das Wort ergreifen, um eine emotionale Explosion zu vermeiden, sondern nur über den anwaltlichen Filter am Prozessgeschehen teilnehmen.

Aber auch das Gegenteil ist möglich, vollendete Noblesse bei härtesten Sachkonflikten. Einem Mehrheitsgesellschafter etwa, der seinen außerordentlich tüchtigen Geschäftsführer wegen allzu hoher, vertraglich für Jahre gesicherter Tantiemenansprüche in einer wirtschaftlichen Stagnationsphase loswerden will, kann daran gelegen sein, alle denkbaren Pflichtverletzungen des Geschäftsführers aufzulisten, damit wenigstens eine übrig bleibt, die vor Gericht Bestand hat und eine fristlose Kündigung trägt. Hier kann es geschehen, dass beide Seiten ihre gegenseitige langjährige Wertschätzung ständig betonen und der Gesellschafter die Kündigung zutiefst bedauert, nur wegen einer - allerdings weitreichenden - unternehmerischen Fehlentscheidung, etwa der tantiemensteigernden bilanziellen Fehlbewertung einer Immobilie, die Vertragsfortsetzung für unzumutbar erklärt. Natürlich wird das Gericht bei so höflichem Umgang miteinander die Zumutbarkeit der weiteren Zusammenarbeit ins Spiel bringen, vor allem wenn es die wirtschaftliche und soziale Relevanz von Umgangsformen überschätzt. Der Prototyp des äußerst liebenswürdigen, aber in der Sache extrem harten Verhandlungspartners war der Unternehmer Neckermann. Von diesem Typ gibt es durchaus noch Exemplare, den gebildeten Weinhändler oder auch den souveränen Zeitungsverleger, der seine Abscheu gegenüber den Widerwärtigkeiten seiner eigenen Postillen durchblicken lässt und diese mit Marktzwängen und dem Profilmangel seiner Nachfrager entschuldigt. Es kann also durchaus sein, dass die Konfliktwirklichkeit unter dem personalen Zivilisiertheitsüberschuss teilweise verdeckt bleibt und dieser womöglich zu Kommunikationsstörungen in der Sache führt. 
Die Anwaltschaft wird nur im Strafprozess genauer beobachtet. Strafverteidiger brauchen Chuzpe gegenüber der staatlichen Autorität, insbesondere der Strafverfolgung. Daraus hat sich bei relativ vielen ein Obstruktivismus à la Bossi entwickelt, der für Mandanten je nach Gericht sowohl zur Straferhöhung führen kann wie zum vorzeitigen Prozessabbruch wegen Unerträglichkeit mit Einstellung des Strafverfahrens. Der Zivilrechtsanwalt muss dagegen im Grundsatz kooperativer auftreten. Die Anwälte bestimmen hier Streitgegenstand und Prozessstoff autonom. Die schriftliche Vorbereitung ist entscheidend, ein schlechtes Verhältnis zum Gegenanwalt ist regelmäßig in der Korrespondenz nicht erkennbar. Die Anwälte diktieren fast ausnahmslos ihre Schriftsätze und sind sich meist des illiteraten Charakters dieser Texte bewusst. Natürlich versuchen sie, den Kontrahenten vielfach auch zu überlisten oder zu übertölpeln. So kann etwa jemand aussichtslos mitverklagt werden, damit er nur als Zeuge ausscheidet. Vielleicht kann ein Kollege auch ganz unmerklich dazu gebracht werden, sich auf etwas einzulassen, was er bei genauer Überlegung ablehnen würde, etwa die Zuständigkeit des Gerichts kraft Einlassung. Persönliche Anfeindung im Schriftverkehr findet schon einmal statt, so wenn im Schriftsatz die Untersuchung der Geschäftsfähigkeit des Gegenanwalts angeregt wird, ist aber ein verhältnismäßig seltener Fremdkörper der Korrespondenz.

In der mündlichen Verhandlung kann aber alles anders werden, vor allem, wenn die Parteien dabei sind. Nun gilt es, sich ins Zeug zu werfen, soweit das Gericht dem eigenen Anliegen nicht folgt. Die persönliche Verschärfung kann von der Behauptung, der Kollege habe Unterlagen unterdrückt, bis zur Kompetenzleugnung reichen, hält sich aber meist in einem solchen Rahmen, dass nachträglich ein kollegiales Grußverhältnis oder gar das vertrauensvolle Miteinander von old boys übrig bleibt, wie das die klassische Karikatur schon bei Daumier gezeigt hat. »Lieber Kollege, Sie werden heute gegen mich genau dasselbe vorbringen, was ich vor drei Wochen in einer ähnlichen Sache plädiert habe, hehehe. Komisch, nicht? - Und ich werde Ihnen dasselbe antworten, was Sie mir damals entgegenwarfen. Das ist amüsant, da können wir uns nötigenfalls soufflieren! « So lautet Daumiers Unterschrift unter einer seiner Lithografien mit dem Dialog zweier Anwälte beim Anziehen der Robe. ${ }^{\text {Is }}$

Das Engagement in der Verhandlung des Zivilprozesses kann natürlich nur etwas bringen, wenn das Gericht noch offen für Argumente und neue Tatsachen ist. Richtet sich die rhetorische Überzeugungsleistung nicht auf die wirtschaftliche Logik, sondern auf eine moralische Dimension, dann kann auch der gegnerische Kollege (auch um seines Mandanten willen) angefasst sein. Es gibt den moralisch begabten Anwalt, der seine Empörung verbal und körpersprachlich expressiv einführen und damit auch jenseits des Rechtsprogramms Punkte machen kann. Das gelingt aber nur bei sparsamer Verwendung und strategischer Problemauswahl. Moralischer Druck kann dann auch zur Einschränkung an sich bestehender Rechte und Pflichten führen.

Auch gesellschaftlicher Druck kann fühlbar werden. Der Anwalt aus renommierter Praxis kann den Einzelanwalt als Kontrahenten womöglich ignorieren, nur mit dem Gericht sprechen und allenfalls zu dem Kollegen über das Gericht. Auch ständiges Unterbrechen ist eine Methode, die Ungleichgewichte schaffen kann, ebenso das Stellen von Fragen, die ein Kollege nicht ohne genaueres Aktenstudium beantworten kann, etwa nach dem Datum einer Mahnung aus dem Jahre 1997. Zivilrichter sehen sich oft ratlos Anwälten gegenüber, die gezielt mit Kommunikationsstörungen

Is $\operatorname{Veth}($ Fn. 2), S. 64. 
gegeneinander arbeiten. Kaum sagt der Kontrahent etwas Substantielles, wird er mit dem Hinweis auf Verspätung konfrontiert oder mit der Behauptung, er habe im Schriftsatz vom Soundsovielten ganz anders vorgetragen, oder gar mit der These, er kenne die Rechtsprechung des Bundesgerichtshofs nicht. Im Regelfall bringt der mündliche Zusatzvortrag aber auch ohne Störung keine entscheidende Wendung. Oft beeindrucken die Anwälte damit nur die eigene Partei, die die Bedeutung der Sprachquanten und der Lautstärke leicht überschätzt. Wie sehr dies den Anwälten bewusst ist, ist auch daraus zu entnehmen, dass sie meist darauf verzichten, irgend etwas von ihren luziden Ausführungen ins Protokoll aufnehmen zu lassen, wo es dem sofortigen Vergessen entwunden und der nächsten Instanz zugänglich gemacht werden könnte.

\section{Die Beziehung der Richter untereinander}

Die Richterschaft ist, wie dargelegt, fast so heterogen wie die Gesellschaft, weswegen bei Zuständigkeit des Einzelrichters für eine Klage schon dessen Haltung bedacht werden muss, etwa bei einer Wohnungskündigung. Auch der Einzelrichter, der nach der letzten ZPO-Reform auch im zweiten Rechtszug vermehrt aktiv ist, ${ }^{16}$ schielt aber in jedem Fall nach der vermutlichen Linie der nächsten Instanz, weil eine Aufhebung seines Urteils weder seiner Karriere noch der bei ihm obsiegenden Partei nützen würde. Die immer größere Dichte der Entscheidungspublikationen erleichtert ihm auch die Anpassung an die herrschenden Meinungen. Wo es keine derartige Orientierung gibt, wird auch der Einzelrichter jedenfalls gelegentlich das Gespräch mit anderen Kollegen während der Vorbereitung einer Rechtssache suchen. Bei kleineren Streitigkeiten wölbt sich, wie man sagt, der blaue Himmel über ihm, weil kein Rechtsmittel gegen seine Entscheidung mehr zulässig ist. ${ }^{17}$ Hier ist er nicht nur in der Verfahrensgestaltung freier, sondern vielfach wesentlich selbstherrlicher. Dann kann sich die mit ihrem Vortrag übergangene Partei nur noch unter Berufung auf Grundrechte, vor allem das Grundrecht auf rechtliches Gehör, wehren.

Die Spruchkörper der Instanzen führen regelmäßig eine wöchentliche Beratung durch. Der Berichterstatter trägt hier aufgrund möglichst genauen Aktenstudiums unter Bezug auf ein schriftliches Votum mündlich vor. Der Vorsitzende, der die mündliche Verhandlung später zu leiten hat, entwickelt aufgrund der Beratung dafür einen Fahrplan. Insgesamt wird ein rechtlicher Konsens in der jeweiligen Sache gesucht. Das Votum des Berichterstatters wird häufiger übernommen als modifiziert. Aber es kommt schon zu gegensätzlichen Wertungen, jedoch äußerst selten zu einer Abstimmung, sofern sich eine Mehrheit im Gespräch stabilisiert. Der Vorsitzende hat regelmäßig die anderen Richter noch in dienstlichen Zeugnissen zu beurteilen, so dass sein hierarchisches Gewicht oft prägend ist. Auf dieser Basis kann er, wenn er so gepolt ist, die anderen intensiv dominieren, insbesondere wenn er deren Urteile bis zur Pensionsgrenze sprachlich, stilistisch und fachlich korrigiert, in der Verhandlung die alleinige Sprecherrolle usurpiert und seinen eigenen Wertungen umfassend Raum verschafft. Wenn er bei einem Verhandlungstermin von einer Stunde weitgehend

I6 Siehe $\ 526$ ZPO, der die Entscheidung über die Berufung gegen eine Einzelrichterentscheidung auf den Einzelrichter des Berufungsgerichts zu übertragen erlaubt, und $\ 5_{27} \mathrm{ZPO}$ zur Vorbereitung der Kollegialentscheidung durch den Einzelrichter.

I7 Aufgrund der Zulässigkeitsvoraussetzung des \5 I I II Nr. I ZPO, die gegenwärtig eine Berufungssumme von mehr als $600 €$ verankert. Zu Streitwerten bis $600 €$ ermöglicht $\$ 495$ a ZPO dem Amtsrichter ein Verfahren nach freiem Ermessen. 
selbst vorträgt und antwortet, bleibt für die anderen Prozessbeteiligten einschließlich der Beisitzer des Gerichts nur eine Statistenrolle.

Das Gegenteil ist derjenige, der von seinen Richterkollegen eine selbständige Prozessleitung erwartet, die Beratungen kurz hält, nur zur mündlichen Verhandlung sicher präsent ist, dort mit allgemeinem Wohlmeinen als eine Art Grüßonkel präsidiert und die Urteilsentwürfe nach ersten Stichproben mit dem jeweiligen Richter jeweils nach kursorischer Lektüre unterschreibt. Schwer zu verarbeiten sind größere fachliche Qualitätsunterschiede innerhalb der Spruchkörper, wobei gesteigerter Fleiß, Milde der kritischen Anmerkungen und kompensative geistige Leistungen, etwa eine bildungsmäßige Anreicherung des Diskurses, relevant sind. Freundschaften entstehen innerhalb solcher Spruchkörper selten, auch wenn die Richter nach einigen Jahren ständiger Beratungen und Verhandlungen vertraut miteinander sind. Dementsprechend entwickelt sich zumindest unter Männern regelmäßig ein burschikoser Ton, wo auf die jeweiligen Schwächen angespielt wird, dass die Kollegen zu unpünktlich, zu verfressen, zu übereifrig, zu ausführlich, zu langsam oder zu radikal sind. Frauen, die zur Zeit weitgehend paritätisch eingestellt werden und deren Anteil in den Beförderungsstufen und Führungspositionen auch ständig steigt, tun sich oft schwer damit, diese kleineren Sticheleien mitzutragen, die zwar für eine heitere Grundstimmung sorgen, aber auf Dauer Verletzungen hinterlassen können. Es ist auch nicht selten, dass ein altbewährter Beisitzer sich nach zehn und mehr Jahren bei seinem Vorsitzenden mit der Bitte meldet, in einen anderen Senat wechseln zu dürfen, wobei die dafür gegebene Begründung regelmäßig Vertuschungscharakter hat.

Es gibt auch Senate mit lauter selbstbewussten Richtern, die bei einem komplexen Rechtsstreit alle unterschiedlicher Meinung sind, dann für die mündliche Verhandlung einen Formelkompromiss finden und in dieser sämtlich zusätzliche Begründungen für ihre Meinung suchen. Der Vorsitzende hat etwa einen Zeugen vernommen, der Berichterstatter vernimmt ihn dann noch einmal etwas nuancenreicher, und der andere Beisitzer versucht, durch Nachfragen die Widersprüche der Zeugenaussage herauszuarbeiten, die seine Kollegen nicht sehen wollen. Parteien und Anwälte können dann hier einhaken. Der Kampf ums Recht kann also innerhalb eines Spruchkörpers nach den Gesetzen der Gruppendynamik sogar dazu führen, dass ein Richter, der schließlich nachgibt, in der nächsten Rechtssache einen Zustimmungsbonus hat. Ganz schwer zu begründen ist es, wenn im Urteilsentwurf eines Kollegen längere Passagen geändert werden sollen. Dann muss eine Alternativformulierung vorgelegt werden. Der Unterlegene wird den vorgezogenen Entwurf dann noch einer detaillierten Korrektur und Verbesserung unterziehen. Daran können langjährige Beziehungen zerbrechen. Diese sind vielleicht auch nicht mehr bei einem Kammeressen zu kitten.

\section{Die Beziebungen des Gerichts zu den Parteien}

Von den Parteien kommt nur Unsinn! Wozu haben wir die Anwälte? Das ist eine verbreitete Alltagsauffassung in der Justiz, die oft dazu führt, dass in den mündlichen Verhandlungen eine Partei erst dann zu Wort kommt, wenn Richter und Anwälte alles gesagt haben, was sie zu allem und jedem sagen wollten, also am Ende der Terminstunde oder erst auf dem Flur. Stellen die Parteien dennoch zu Beginn eine Verständnisfrage, wird ihnen dann bedeutet, dass ihre Anwälte ihnen alles erklären können. So beschränkt sich vielfach die Rolle der Parteien auf die Assistenz für den eigenen Anwalt, dem zugeflüstert werden kann, was am Tatsachenvortrag des 

nachtragen, heißt es schnell: »Das steht schon in den Akten! Würden die Gerichte dafür sorgen, dass auch die Parteien ihre Sicht wenigstens einmal noch kompakt vortragen können, käme es wahrscheinlich zu einer Vermehrung derjenigen Prozesse, wo plötzlich ein Streit überraschend endet. Das Szenario dafür: Die Partei erklärt noch einmal eindringlich, warum ihr Anspruch begründet oder der Gegenanspruch unbegründet ist. Sie hat aber auch Verständnis, dass das Gericht ihr nicht ganz folgt. Sie bedankt sich dafür, dass ihr jedenfalls einmal ein Gericht wirklich zugehört hat, und nimmt ihre Klage, die Rechtsmittel, ihren Widerspruch, ihren Einspruch, ihre Beschwerde wenigstens halbzufrieden zurück.

Die Rolle der Parteien wird bedeutsamer, wenn das Gericht dem Anwaltsvortrag keinen realen Glauben schenkt. »Haben Sie wirklich nach der zahnärztlichen Behandlung zwei Monate lang solche Schmerzen gehabt, dass Sie nicht arbeiten konnten? « kann eine einfühlsame Frage im Arzthaftungsprozess lauten, wenn eine anwaltliche Überhöhung des Schmerzensgeldanspruchs im Schriftsatz vermutet wird. Der Kläger, der den Schriftsatz so genau nicht im Kopf hat, sagt dann etwas, was vielleicht immer noch übertrieben ist, aber doch nicht in dem Maße wie durch den Anwalt, worauf sich der gesamte Geschehensablauf anders darstellen kann. Ebenso wie Schmerzen können sich die Frustrationen einer missglückten Reise, die Beteuerungen eines Anlageberaters, die anderweitigen Einsatzmöglichkeiten eines vor verschlossener Tür stehenden Bauhandwerkers, die Belästigungen durch Prostitution in der Nachbarschaft und andere Leiden als prozessbezogene Dramatisierungen erweisen, die mit knappen Protokollsätzen zu korrigieren sind.

Vor allem aber braucht das Gericht die Parteien für eine gütliche Einigung durch Vergleich. Hier können unterschiedliche Resistenzen von Anwalt und Partei anzutreffen sein. Zur Sprache kommen können etwa die Leistungsfähigkeit, um einen realitätsgerechten Tilgungsplan zu konkretisieren, die Versicherungssituation, die Einbeziehung Dritter, Teilerlasse und Sofortleistungen, vor allem aber auch die Prozesskostensenkungen. Damit können Lösungen möglich werden, bei denen der Anwalt dann sagt, er wisse nicht, ob er seiner Partei eine solche Einigung empfehlen könne. Wahrt diese aber beiden Parteien das Gesicht, ist sie praktikabel, erspart sie dem Gericht den Urteilstext und allen Beteiligten die Inanspruchnahme weiterer Instanzen, können auch die Anwälte nicht mehr dazwischen funken, selbst wenn es für sie dann nichts mehr zu verdienen gibt. Wo aber eine Einigung der Parteien völlig aussichtlos ist, zum Beispiel mit einer Versicherung, die ein Urteil als Steuerungsgrundlage braucht, kommen die Parteien kaum zum Zug. Bestenfalls werden ihnen, wenn das Gericht schon seiner Entscheidung sicher ist, Empfehlungen zur Klagrücknahme oder zur Anerkennung des Anspruchs gegeben.

\section{Die Beziebungen des Gerichts zu den Anwälten}

Die Gerichte versuchen, sich, wo es geht, eine Vorstellung davon zu machen, wie die Anwälte sich beim Rechtsgespräch verhalten werden, ob sie renommiert, fachlich beschlagen, hartnäckig, flexibel, entscheidungsfähig, zickig, locker oder nichts von alledem sind. Erscheinen keine Parteien im Termin, so kann die informelle Kommunikation entre nous geradezu dominieren. Ein Beispiel aus der zweiten Instanz: »Welche Freude, dass ein so viel beschäftigter Anwalt wie Sie mit all seinen Ehrenämtern sich extra aus ... zu einer so bescheidenen Sache mit kleinem Streitwert bei uns einfindet! « kann einem Anwalt entgegengerufen werden, bevor er im Gerichtssaal 
Platz nimmt. »Oder sind Sie nur auf der Durchfahrt in die Toskana?« kann die Fortsetzung sein, wenn er geschmeichelt lächelt. Der Anwalt tritt etwas näher an den Richtertisch, sagt zu dem ungläubig dreinblickenden Referendar: »Ich werde hier immer beleidigt « und zum Vorsitzenden lächelnd: »Ich traue der Rechtsfindung des Senats zu wenig, als dass ich einen Mitarbeiter schicken könnte.« Es wird weiter gefrotzelt, als der Gegenanwalt erscheint. Der Vorsitzende: »Was haben Sie für eine schöne Krawatte. Können Sie sich diese bei solchen Ministreitwerten überhaupt leisten?« »Die hat mir meine Frau geschenkt.«»Ihre Frau hat Geschmack! « Dann beginnt die Erörterung des Rechtsstreits. Der Prozessbevollmächtigte des klagenden Bauunternehmers verlangt hier ein Zusatzentgelt für Zeugnisse hinsichtlich einwandfreier Meßeinrichtungen zum Zweck des Nachweises bei staatlichen Behörden, der Bauherrenvertreter meint, diese Kosten seien von dem vereinbarten Pauschalpreis abgedeckt. Das Gericht hält die Forderung nur teilweise für berechtigt, ein weiterer Teil der Forderung ist noch in erster Instanz anhängig. »Sie wollen doch wegen dieses Krams nicht noch einmal aus ... kommen? « wird der eine Anwalt angepflaumt. Dem anderen wird geraten: »Sie sollten mehr Zeit mit ihrer netten Frau verbringen.« Ein letzter Versuch des Widerstands: »Mein Mandant nimmt jede kleine Position sehr ernst. « "Sie werden ihn schon trösten. « Es wird unter spürbarem Druck ein widerruflicher Vergleich über alle Streitpunkte, auch die der ersten Instanz, geschlossen. »Wehe, Sie widerrufen! Dann setzen wir die Sache in aller Frühe an.« Man winkt sich zum Abschied ironisch zu.

Bei existentieller Notlage kann sich solcher Schalk natürlich verbieten. Aber auch bei kleineren Konflikten verpflichtet die Anwesenheit der Parteien im allgemeinen zu gewissenhafter Erörterung selbst des Minderwerts defekter Teppichleisten. Zu erwähnen sind Vorsitzende, die hier sicherheitshalber stereotyp beteuern, man habe alles »nach bestem Gewissen« geprüft. Die Anwälte werden aber recht differenziert behandelt. Manchen hört man gar nicht zu, und die Betroffenen wagen sich in Gegenwart ihrer Mandanten nicht einmal zu beschweren. Bei anderen wird jedes Mikroargument aufgegriffen und als beachtlich bezeichnet. Hat ein respektierter Anwalt in einer Rechtssache schlechte Karten, wird der Partei schon einmal versichert: »Ohne die ausgezeichnete Arbeit Ihres Anwalts wäre längst alles gegen Sie entschieden.« Das stärkt dessen Position für den Prozessverlust. Natürlich spielt es auch eine Rolle, ob ein Anwalt regelmäßig eine halbseidene Klientel vertritt oder konventionelle Bürger. Die Parteien zu vergleichen, bedeutet ferner stets, dass sich aus dem Prozess auch für die Anwälte ein befriedigendes wirtschaftliches Resultat ergibt, ohne dass dies den Parteien bewusst werden darf. Die darauf gerichtete Kommunikation mit den Anwälten wird unter Umständen leichter gelingen, wenn sie an einem Stehpult hinter dem Mandanten im Gerichtssaal stehen.

Die Anwälte haben es ferner zu berücksichtigen, wenn sie vor dem Gericht immer wieder Prozesse zu führen haben, also keine verbrannte Erde hinterlassen dürfen. Allzu große Kompromissfähigkeit kann andererseits abträglich sein. Am besten ist der Status des Anwalts, bei dem das Gericht schon einmal auf Granit gebissen hat, dem danach aber wieder die Kooperation gelungen ist. Insofern kann auch eine aussichtslose Verfassungsbeschwerde wegen Grundrechtsverletzung oder ein zurückgewiesener Befangenheitsantrag als Unannehmlichkeit ein Respekt begründender Erinnerungswert sein.

Die Beziehungsstrukturen der Prozessbeteiligten können hier nicht umfassend behandelt werden. Der weiteren Untersuchung anheimzugeben sind insbesondere auch die Beziehungen zwischen den Anwälten und ihren Zeugen und Sachverständigen. Aber auch die bisherige Analyse zeigt ein Geflecht von Interessen-, Macht- und Zuwendungsverhältnissen, das dem rein normativen juristischen Betrachter als ein 
Ensemble von ungeheuren Defiziten gegenüber den rechtsstaatlichen Geboten erscheinen könnte. Dies wäre jedoch ein grundlegendes Missverständnis der rechtspsychologischen Befunde. Vielmehr schließen es die hier erörterten Besonderheiten der zivilprozessualen Kommunikation keineswegs aus, dass die Mehrzahl der Beteiligten durchaus verantwortungsbewusst handelt. Die Kommunikationsstrukturen lassen sich keineswegs grundlegend ändern. Wer vor oder hinter den Schranken des Gerichts steht, sollte sich nur dieser Strukturen bewusst sein, um mit ihnen umgehen zu können.

\section{Fazit}

Versteht man Rechtspsychologie anhand des hier ausgebreiteten Materials zu den forensischen Strukturen des Zivilprozesses als Psychologie rechtlich formulierter Befriedungsprozesse, dann wird ihr Programm sein, die Bedingungen psychischer Internalisierung der ökonomischen und sozialen Prozesse und ihrer rechtlichen Eingrenzung zu präzisieren. Eine empirische Forschungsbarriere besteht allerdings darin, dass die Justiz ihre Entscheidungstätigkeit gegenüber der unmittelbaren Teilhabe von Forschern abschirmt. Teilnehmende Beobachtung ist nur den Mitgliedern des Justizsystems möglich, die aber eine Explorierung des internen Entscheidungsgangs und den Mitschnitt des Rechtsgesprächs der Verhandlungen mit ihrer Verabschiedung quittieren müssten. Auch Aktenlektüre scheitert am Individualrechtsschutz. Da werden nur die Rezeptionen von der Hinterbank der Öffentlichkeit aus, die Auswertung der publizierten Entscheidungen und Interviews mit den Beteiligten weiterhelfen. Auch die Mediationslehre versucht, eine selbständige Befriedungsleistung zu konstruieren und $\mathrm{zu}$ organisieren. Das Zivilgericht ist dafür als Zwangsanstalt zwischen Klage und Vollstreckung aber nur begrenzt offen. Rechtspsychologie muss sowohl die Freiräume der Konsensfindung innerhalb der rechtlichen Sachzwänge erkunden wie auch die Umsetzung unvermeidbarer ökonomischer und sozialer Imperative stützen. Nicht zuletzt kann sie aber auch das Unrecht zu verarbeiten helfen, das bei den nach menschlichem Maß unvermeidbaren Fehlleistungen der Justiz laufend anfällt. Was der normativ inspirierte Jurist aufgrund der erörterten Rechtstatsachen und Beziehungsstrukturen als Defizite der gegenwärtigen Verfassung des Zivilprozesses werten, eventuell auch leugnen oder pointieren wird, ist denn auch nicht einfach durch Gesetzesnovellen zu ändern. Vielmehr bedarf es einer die Prozessrechtsnormen übergreifenden Reflexion, auch gerade unter rechtspsychologischem Aspekt, welche allzumenschlichen internen und externen Kommunikationsverhältnisse der Zivilgerichte dann doch mit den Prinzipien der Rechts- und Sozialstaatlichkeit nicht zu vereinbaren sind. 